\title{
Research on Fast Face RecognitionAlgorithm Based on Block CS-LBP and HIK Kernel Method
}

\author{
Shaoming Pan, Gongkun Luo, Baozhong Ke and Kejiang Li* \\ School of Electrical and Information Engineering, Guangxi University of Science \\ and Technology, Liuzhou, Guangxi, China, 545006 \\ 23945052@qq.com
}

\begin{abstract}
With the development of artificial intelligence and pattern recognition technology, more and more research related to human face is constantly developing in all walks of life. At the present stage, the traditional face recognition algorithm based on LBP and SVM is not good, and the process of feature extraction and feature classification are deeply studied in this paper. For feature extraction, the authors put forward an improved CS-LBP texture feature; for feature classification, the author uses the histogram intersection (HIK) kernel function to classify the features which has high efficiency and good effect. Subsequently, experiments are carried out on the Yale data set and the ORL data set. Experimental results show that the proposed algorithm has a significant improvement on the face recognition effect of face direction change, and the illumination change is slightly improved. In the natural environment, most face recognition has the influence of human face direction and noise, and the effect of noise is a hot direction of face recognition research in the future.
\end{abstract}

Keywords:Face recognition; CS-LBP texture; Support vector machine; HIK kernel

\section{Introduction}

Face recognition [1] is a hot subject in the fields of biology and computer science. Differentfrom face detection, face recognition is for face image authentication. It determines each image belongs to which people, while face detection [2] is the judgment of the image, whether there is a face in the image. With the development of computer intelligence technology, face recognition and authentication in most areas have been widely used, such as criminal investigation, forensics and the latest face attendance and so on. Because the face image is a rigid object image which has a good geometric characteristics. So face recognition is easier compared to recognition of non-rigid objects, and there are many researches on face recognition.

Face recognition is a kind of pattern recognition, which usually has two stages: feature extraction and feature classification. Because of the particularity of the face image, the most commonly used feature is texture feature. The most popular features include LBP texture features [3], Gabor texture features [4] and so on. After extraction of image features, characteristics of face images replace the original image. Then the classification algorithm of machine learning is used to classify the different faces into the corresponding categories. Common classification methods including support vector machine (SVM) [5], artificial neural network (NN) [6], the logistic regression (LR) [7] and so on, different machine learning methods have achieved good results in their respective fields.

In this paper, by studying the shortcomings of the LBP texture feature, the original feature is replaced by the improved CS-LBP texture feature. In the classification process,

${ }^{*}$ Corresponding Author 
the author chooses support vector machine classifier which is the fastest and the most suitable for face recognition, and improves the existing support vector machine by means of histogram intersection kernel function. Some accuracy is improved on the result of face recognition. Inaddition, the support vector machine of the histogram cross kernel function can converge faster to improve the efficiency of face recognition.

\section{Algorithm for Feature Extraction in Face Recognition}

\subsection{LBP Texture and Feature Algorithm of Improved Texture}

Local binary pattern (LBP) is a local texture feature descriptor. The descriptor generates a descriptor for each pixel through the relationship between each pixel and its neighboring pixels, the descriptor can show the local texture features around the pixel.The simplest LBP operator is to calculate the difference between the gray value of the current pixel and the surrounding 8 neighborhood,then local LBP operators is obtained bythe difference value of two.There are many improvements to the traditional LBP algorithm version on this basis.

The localization of the traditional LBP algorithm is more serious, and it is easy to be affected by the local noise. Under normal circumstances, the local noise will strengthen or weaken the texture intensity between the region and the surrounding area.Because of this, people put forward the concept of "uniform pattern" [8]. The theory thinks that in the actual image, a lot of common mode is the basic attributes of texture, it can contain and express mosttexture features. These jump mode that only contain two times from 1 to 0 or 0 to 1 are called equivalent model, these equivalent models can represent the texture features of most of the actual image, and take a high proportion. Therefore, the application of Uniform-LBP is relatively wide, the texture feature of original high dimensional is reduced to a lower jump level, which enhance the efficiency of the extraction of texture features.

In addition, in order to effectively preserve the edge and contour information of the original image in the process of calculating the texture feature, we propose a method for computing the CS-LBP texture feature [9]. CS-LBP texture feature redefines the comparison rules between the values of the pixels. The traditional way is to compare the pixel gray value with the center of the pixel value, and the improved method is that it is compared with the pixel value of center symmetry. By comparing the difference between the gray values of the pixel value, the method can combine the two advantages of the texture feature extraction and the gradient calculation method of the LBP operator, which can preserve the edge and contour information in the texture shape. The following figure gives the extraction methods and processes of above three kinds of texture feature. In the process of face recognition, due to the contour of the face edge information in face recognition can play a significant role, so choose CS-LBP texture feature extraction algorithm and improve it, so that it can enhance the recognition rate in face recognition.

\begin{tabular}{|l|l|l|}
\hline $\mathrm{g} 0$ & $\mathrm{~g} 1$ & $\mathrm{~g} 2$ \\
\hline $\mathrm{g} 7$ & $\mathrm{gc}$ & $\mathrm{g} 3$ \\
\hline $\mathrm{g} 6$ & $\mathrm{~g} 5$ & $\mathrm{~g} 4$ \\
\hline
\end{tabular}

$L B P_{8,1}=\sum_{p=0}^{7} S\left(g_{p}-g_{c}\right) \mathbb{2}^{p}, S(x)=\left\{\begin{array}{l}1, x \geq 0 \\ 0, x \leq 0\end{array}\right.$ 


$$
\begin{aligned}
& L B P_{8,1}^{v}=\left\{\begin{array}{l}
I(L B P), U(L B P) \leq 2 \\
58, \text { otherwise }
\end{array}, I(z) \in[0,57]\right. \\
& U(L B P)=\left|S\left(g_{7}-g_{c}\right)-S\left(g_{0}-g_{c}\right)\right|+\sum_{p=1}^{7}\left|S\left(g_{p}-g_{c}\right)-S\left(g_{p-1}-g_{c}\right)\right| \\
& C S-L B P_{8,1}=\sum_{p=0}^{4} S\left(g_{p+4}-g_{p}\right) \mathbb{2}^{p}, S(x)=\left\{\begin{array}{l}
1, x \geq T \\
0, x \leq T
\end{array}\right.
\end{aligned}
$$

\subsection{Texture Features of 2D CS-LBP}

One dimensional CS-LBP can only better reflect the texture and contour features of the original image, which cannot be a good response to the image of the gray space correlation. On this basis, this paper proposes a two-dimensional CS-LBP texture feature, which is divided into two dimensions. The first dimension is the computation of CS-LBP texture. Second dimensions are used to represent spatial correlation. This paper extracts the CS-LBP texture features of the four directions: vertical direction, the horizontal direction, positive 45 degree and negative 45 degree. The real feature of face image is represented by the texture feature, which can better show the characteristics of local texture, and because of the strengthening of the spatial correlation, it can fundamentally inhibiting effect brought by the local noise on CS-LBP texture. Assuming that $\delta$ is a one dimensional CS-LBP texture feature, the two dimensional CS-LBP features can be represented as:

$$
P(\text { angle }, \delta)=\left\{\delta_{0}, \delta_{45}, \delta_{90}, \delta_{135}\right\} \quad(2-1)
$$

Through the calculation of two-dimensional CS-LBP features, it can better express relevance of texture features in the spatial correlation, because in the face recognition, the geometric relationship between texture features plays an important role in the process of identifying face.

\subsection{Texture Feature of Block 2D CS-LBP}

Previous section analyses the deficiencies of the traditional LBP, and introduces CS-LBP features who has a betterrobustness. It has strong anti-interference ability, and retains the contour features of the original image, can better complete the task of classification and recognition. In addition, in order to obtain the relationship of facial texture features in geometry. Thispaper extracts 2D MB-LBP texture based on the feature of CS-LBP. This feature is not only robust to noise robustness, but also has the invariance of texture rotation geometry, which will be better for face recognition.

Face recognition experiment is carried on based on CS-LBP+SVM classifier (see section fourth).The experimental results show that the recognition rate is not obvious, so on this basis, this paper proposes a block CS-LBP texture features for face recognition.In general, as the number of features is too small to calculate CS-LBP histogram by whole image, thedistribution results are not obvious. For the face recognition results, 2D CS-LBP features can provide more feature dimension and characteristics of diversity, and the effect will be better. Firstly, the face images are divided into $2^{2 N}\left(2^{N} \times 2^{N}\right)$ sub blocks after CS-LBP feature extraction, where $N$ representation block series, it computes the histogram for CS-LBP features of all sub blocks in the $N$ level block. Cascading sub block histogram can get a set of image features. For four directions in two-dimensional, calculating texture feature of block CS-LBP according to the above operation, and finally get tithe texture features include not only the contour information of CS-LBP, but also the geometric rotation characteristics of two-dimensional features, and 
the diversity of texture after block. We can gradually improve the characteristics of the texture features through three steps; these features will play an important role in the face recognition.

To sum up, the process of the method is as follows:

1. To calculate the two-dimensional center symmetric CS-LBP texturein the original image, and save the texture feature map from four directions;

2. 4 CS-LBP texture feature map is divided by a given series $\mathrm{N}$, the experiment obtains different recognition rate through different blocks, and the block with highest recognition rate will be selected;

3. Calculate the histogram of each small region after the block, give the texture features of final block two-dimensional CS-LBP through the statistical histogram.

The following Figure 1, is extraction flow chart of texture feature of improved block 2D CS-LBP.

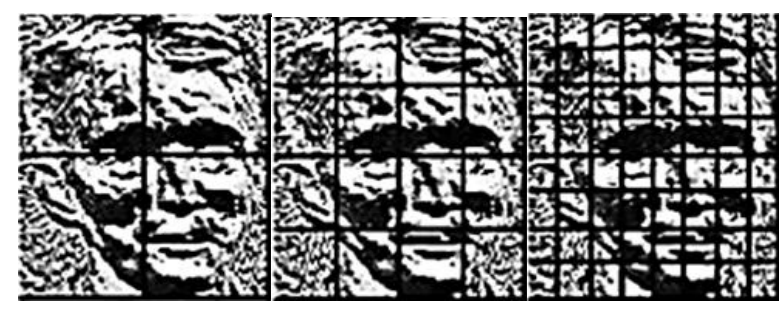

$\mathrm{N}=1$

$\mathrm{N}=2$

$\mathrm{N}=3$

(a) Unblocking images

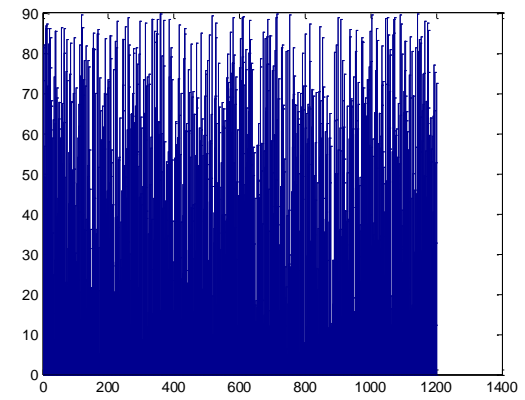

(b) Statistical histogram characteristics for $\mathrm{N}=3$ block

Figure 1. Block and LBP Statistical Histogram

\section{Classification Algorithm for Face Recognition Feature}

\subsection{Support Vector Machine}

Support vector machine (SVM) [10] developed from the perceptron,traditional perception machine [9] separate the data by solving linear classification. The data distribution is different, and the linear classification is more, but the support vector machine needs to find an optimal classification surface, so that the data to be classified can be separated from the maximum "interval". Traditional support vector machines are mainly aimed at the binary classification. A large number of studies show that multi category classification problem can be simplified into multiple binary classification problems. In order to solve the image annotation problem, we only need to create multiple support vector machine classification.

Assume that the training $\operatorname{set}\left\{x_{i}, y_{i}, \mathbf{1}=1,2,3 \ldots \mathrm{n}\right\}$ represents a data set, Datax is the sample data needed to classify, while is corresponding label of each data. It is also a description that which category the data needs to be classified. Support vector machines need to find an optimal classification face $\mathrm{w}^{*} \mathrm{x}+\mathrm{b}=0$, so that the sample data set $\mathrm{x}$ can be separated as large as possible. As shown in Figure 2, we need to separate the two 
categories of data as far as possible, which is the best classification shown in the graph.

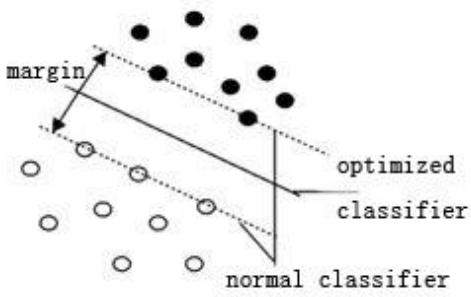

\section{Figure 2. Support Vector Machine and Optimal Classification Face}

By analyzing the optimal classification surface $w^{*} x+b=0$, where $w$ is the weight, $x$ is the support vector, and $b$ is the additive bias. Calculating the classification face of optimization is also the calculation of two plans for $\mathrm{w}$ and $\mathrm{b}$ :

$$
\begin{aligned}
& \min _{\omega, b} \frac{1}{2}\|\omega\|^{2}(3-1) \\
& \text { s.t. } y_{i}\left(\omega\left[x_{i}+b\right) \geq 1, i=1,2, \ldots, n(3-2)\right.
\end{aligned}
$$

By solving the optimization problem of the two programming, the optimal weights $\mathrm{w}$ and $b$ can be solved by the Lagrange multiplier method and the KKT condition, and then the optimization results of the sample classification are obtained.

\subsection{Kernel Method}

Actually, almost all practical problems cannot meet linearly separable data set $\mathrm{x}$. linearly separable data set cannot just use the linear classification to distinguish them. On the basis of the support vector machine, adding the slack variables, and allowing partial sample emerge errors, in this case we can use the support vector machine method for solving nonlinear separable data sets. In addition, as shown in Figure 3, such a data set cannot be solved through the slack variables. Because if it is classified in accordance with the linear classification plane,then there will be more wrong samples, which leads to the poor performance of the final classifier. At this time, the support vector machine uses the concept of kernel function [11].The original linear non separable data sets can be changed into the high dimension space through the kernel function, and the original data is transformed into a linear separable data set in high dimensional space through high dimensional space mapping. In this way, we can use the linear classification to classify.

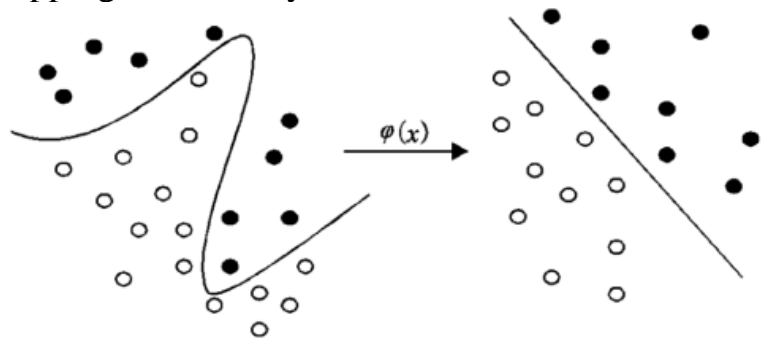

\section{Figure 3. Nonlinear Data Sets can be Divided into High Dimensional Linear Separable Data Sets by Kernel Function}

Among them, $\varphi_{x}$ is the kernel function and we can transform the two optimization problem of support vector machine to the following:

$$
\min _{\omega, b} \frac{1}{2}\|\omega\|^{2}+C \sum_{i=1}^{m} \xi_{i}
$$




$$
\begin{aligned}
& \text { s.t. } y_{i}\left(\omega \varphi\left(x_{i}\right)+b\right) \geq 1-\xi_{i}(3-12) \\
& \xi_{i} \geq 0, i=1,2,3, \ldots, n(3-14)
\end{aligned}
$$

$\xi_{i}$ is a relaxation factor, $\varphi_{x}$ is the kernel function, and $\mathrm{C}$ is the penalty factor. In the same, we can use the Lagrange multiplier method and the KKT condition to get the optimal solution of the two programming, and then show the classification surface of the nonlinear support vector machine:

$$
f(x)=\operatorname{sgn}\left[\sum_{i, j=1}^{m} a_{i} y_{i} K\left(x_{i}, x_{j}\right)+b\right](3-14)
$$

$K\left(x_{i}, x_{j}\right)$ is a kernel function, we can easily use the support vector machine with kernel function to complete the regression problem of image annotation frame by using kernel function.

\subsection{HIK Nuclear Method}

So far, there is no theoretical study to show that what kind of kernel function is suitable for what kind of categorical data. In general, people use the way of attempt-feedback,selectdifferent kernel functions to test their own data sets, and then choose the best kernel function to do the work of SVM classification.Common kernel functions include the following 4 kinds:

(1) Linear kernel function: $f(x)=\operatorname{sgn}\left[\sum_{i=1}^{n} a_{i} y_{i}\left(x_{i} \square x\right)+b\right]$

(2) Polynomial kernel function: $f(x)=\operatorname{sgn}\left\{\sum_{i=1}^{n} a_{i} y_{i}\left[\left(x_{i}[x)+1\right]^{d}+b\right\}\right.$

(3) Radial basis function: $f(x)=\operatorname{sgn}\left\{\sum_{i=1}^{n} a_{i} \exp \left\{-\frac{x_{i}-x}{\sigma^{2}}\right\}+b\right\}$

(4) Sigmoid excitation function: $f(x)=\operatorname{sgn}\left\{\sum_{i=1}^{n} a_{i} \tanh \left[\gamma\left[\left(x_{i}[x)+\theta\right]+b\right\}\right.\right.$

In general, radial basis functions are often used to classify the dimension of unknown samples, and the original dimension is projected into the infinite dimension, which is the best way to support the LBP texture. At the present stage, the histogram intersection kernel method (HIK) [13], which is proposed by the pattern recognition Laboratory in Nanjing University, has a good classification effect on the high dimensionality of the histogram. The statistical histogram of each block is presented as a feature of face recognition in this paper, which is based on the block CS-LBP method. Therefore, this part selects HIK kernel method for face recognition. Histogram cross kernel method is different from the common four ways, its kernel function is:

$$
\left.f(x)=\sum_{i=1}^{N} \min \left(|x(i)|^{\beta},|z(i)|\right)^{\beta}\right\}, \beta>0
$$

According to the support vector machine, the classification function is defined as:

$$
h(x)=\sum_{l=1}^{m} \alpha_{l} \beta_{l} k\left(x, x_{l}\right)+b, w=\sum_{l=1}^{m} \alpha_{l} y_{l} x_{l}
$$

Among them, $x$ is a two-dimensional CS-LBP texture feature vector, the dimension is $n, x_{l}: l \in\{1,2, \ldots, m\}$ is the corresponding support vector. The corresponding cross 
core can be defined as:

$k(x, z)=\sum_{i=1}^{n} \min (x(i), z(i))$

According to the Formula (3-7), (3-8), (3-9), it can conclude the classification function of SVM classification:

$h(x)=\sum_{i=1}^{n}\left(\sum_{l=1}^{m} \alpha_{l} \beta_{l} \min \left(x(i), x_{l}(i)\right)\right)+b$

The classification kernel and classification function are classified according to the above definition, because the kernel uses the histogram cross kernel method, this method is more efficient for the higher dimension of the histogram.

\section{Realize of Face Recognition System}

\subsection{The Process of Face Recognition System}

Face recognition is a complex nonlinear classification problem. First of all, you need a more reasonable face feature to replace the original image, use low dimensional feature to replace the high dimension of the original image to achieve the purpose of dimension reduction, which can reduce the time complexity of the SVM recognition process. In this paper, the adaptive weight of the mixed kernel function is calculated by the CS-LBP features of the block, and the function of the face recognition is completed by the SVM of the histogram cross kernel function. Face recognition process as shown in Figure 4.

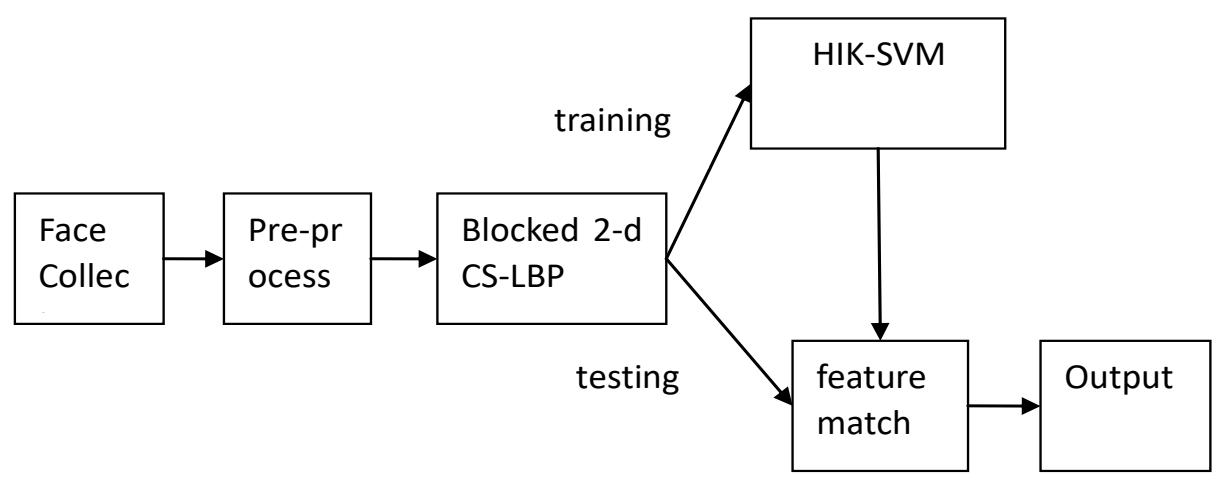

Figure 4. Algorithm for Face Recognition Process

\subsection{Selection of Data Sets}

In this paper, we respectively make a face recognition experiment on ORL dataset and Yale dataset [14-15]. ORL data set is a face data set of University of Cambridge, the data set includes 40 individuals, the illumination intensity of the 10 face images of each person is almost the same, but the angle of the human face is changed. In contrast, the Yale University maintains the face data set with 48 individuals, each has 55 face images. The face image database changes in the light of each image, and the perspective of the human face is almost no change. Asshown in Figure 5, we can see the example of two database face images.

ORL and Yale database have their own advantages. It has a strong horizontal comparison through the two databases on the test of this algorithm. In addition, in this paper, the algorithm is compared with one dimensional CS-LBP, 2D CS-LBP and block CS-LBP texture features in the radial basis function kernel method $k_{R B F}$ and the 
histogram cross kernel method $k_{H I K}$. The experimental environment is carried out on MatlabR2012a.

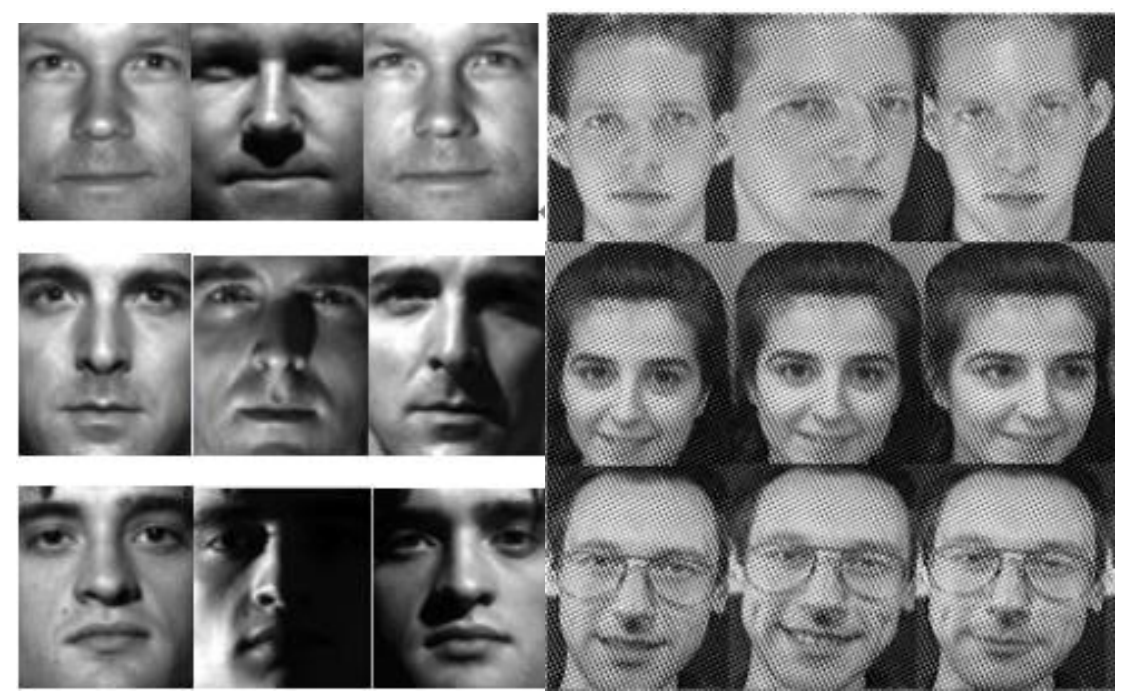

Figure 5. Yale Data Set (Left) and ORL Data Set Image (Right)

\subsection{Comparison of the Experimental Process}

In order to compare the practical significance of the algorithm in the horizontal and vertical, this paper randomly selects 30 individual data sets from the two databases of ORL and Yale, each person has 10 sample images, 7 of which as the training data set and the other three images as the test data set. All samples are normalized to the size of $32 * 32$.

Feature extraction: For the 300 databases of two samples, each sample are extracted from one dimensional CS-LBP texture features, two-dimensional CS-LBP texture features and block two-dimensional CS-LBP features. Among them, the author uses three ways $(\mathrm{N}=2,4,6)$ to do horizontal comparison.

Classification of SVM: SVM classification is carried out for above two kinds of characteristics, in order to horizontal comparison, this paper uses $K_{\mathrm{RBF}}$ and $\mathrm{K}_{\mathrm{HIK}}$ nuclear method for comparison.

\subsection{Experimental Results and Analysis}

12 groups of experiments were carried out according to the above experimental pretreatment and parameter selection, 6 identical experiments were performed on ORL and Yale database. One dimensional CS-LBP feature $+\mathrm{K}_{\mathrm{RBF}}$ kernel function,one dimensional CS-LBP feature $+\mathrm{K}_{\mathrm{HIK}}$ kernel function,two dimensional CS-LBP feature $+\mathrm{K}_{\mathrm{RBF}}$ kernel function,two dimensional CS-LBP feature $+\mathrm{K}_{\mathrm{HIK}}$ kernel function,block two dimensional CS-LBP feature $+\mathrm{K}_{\mathrm{RBF}}$ kernel function and block two dimensional CS-LBP feature $+\mathrm{K}_{\mathrm{HIK}}$ kernel function. The experimental results are given by the following Table 1, and Table 2: 
Table 1. Recognition Rate and Time of Various Algorithms on Yale Data

\begin{tabular}{|c|c|c|c|c|}
\hline Features & Classifier & $\begin{array}{c}\text { Error-classifying } \\
\text { samples }\end{array}$ & Recognition rate & $\begin{array}{l}\text { Recognition } \\
\text { time }\end{array}$ \\
\hline $\begin{array}{c}\text { One } \\
\text { dimensional } \\
\text { CS-LBP } \\
\text { feature }\end{array}$ & $\begin{array}{c}K_{R B F} \text { kernel } \\
\text { function }\end{array}$ & 96 & $68 \%$ & $1.362 \mathrm{~s}$ \\
\hline $\begin{array}{c}\text { One } \\
\text { dimensional } \\
\text { CS-LBP } \\
\text { feature } \\
\end{array}$ & $\begin{array}{l}K_{H I K} \text { kernel } \\
\text { function }\end{array}$ & 75 & $75 \%$ & $1.476 \mathrm{~s}$ \\
\hline $\begin{array}{c}\text { Two } \\
\text { dimensional } \\
\text { CS-LBP } \\
\text { feature } \\
\end{array}$ & $\begin{array}{l}K_{R B F} \text { kernel } \\
\text { function }\end{array}$ & 42 & $86 \%$ & $1.525 \mathrm{~s}$ \\
\hline $\begin{array}{c}\text { Two } \\
\text { dimensional } \\
\text { CS-LBP } \\
\text { feature } \\
\end{array}$ & $\begin{array}{l}K_{R B F} \text { kernel } \\
\text { function }\end{array}$ & 90 & $70 \%$ & $1.253 \mathrm{~s}$ \\
\hline $\begin{array}{c}\text { Block two } \\
\text { dimensional } \\
\text { CS-LBP } \\
\text { feature }(\mathrm{N}=2 \text {, } \\
4,6)\end{array}$ & $\begin{array}{l}K_{R B F} \text { kernel } \\
\text { function }\end{array}$ & $(66,60,57)$ & $\begin{array}{c}(78 \%, 80 \%, \\
81 \%)\end{array}$ & $1.352 \mathrm{~s}$ \\
\hline $\begin{array}{c}\text { Block two } \\
\text { dimensional } \\
\text { CS-LBP } \\
\text { feature }(\mathrm{N}=2, \\
4,6)\end{array}$ & $\begin{array}{l}K_{H I K} \text { kernel } \\
\text { function }\end{array}$ & $(28,25,24)$ & $\begin{array}{c}(90.6 \%, 91.6 \%, \\
92 \%)\end{array}$ & $\begin{array}{l}(1.442 \mathrm{~s}, \\
1.468 \mathrm{~s}, \\
1.497 \mathrm{~s})\end{array}$ \\
\hline
\end{tabular}

Table 2. Recognition Rate and Time of Various Algorithms on ORL Data

\begin{tabular}{|c|c|c|c|c|}
\hline Features & Classifier & $\begin{array}{c}\text { Error-classifying } \\
\text { samples }\end{array}$ & Recognition rate & $\begin{array}{c}\text { Recognition } \\
\text { time }\end{array}$ \\
\hline $\begin{array}{c}\text { One } \\
\text { dimensional } \\
\begin{array}{c}\text { CS-LBP } \\
\text { feature }\end{array}\end{array}$ & $\begin{array}{c}K_{\text {RBF }} \text { kernel } \\
\text { function }\end{array}$ & 144 & $62 \%$ & $1.372 \mathrm{~s}$ \\
\hline $\begin{array}{c}\text { One } \\
\text { dimensional } \\
\text { CS-LBP } \\
\text { feature }\end{array}$ & $\begin{array}{c}K_{\text {HIK }} \text { kernel } \\
\text { function }\end{array}$ & 87 & $71 \%$ & $1.442 \mathrm{~s}$ \\
\hline $\begin{array}{c}\text { Two } \\
\text { dimensional } \\
\text { CS-LBP } \\
\text { feature }\end{array}$ & $\begin{array}{c}K_{\text {RBF }} \text { kernel } \\
\text { function }\end{array}$ & 51 & & $1.534 \mathrm{~s}$ \\
\hline $\begin{array}{c}\text { Two } \\
\text { dimensional } \\
\text { CS-LBP } \\
\text { feature }\end{array}$ & $\begin{array}{c}K_{\text {RBF }} \text { kernel } \\
\text { function }\end{array}$ & 78 & & \\
\hline $\begin{array}{c}\text { Block two } \\
\text { dimensional }\end{array}$ & $\begin{array}{c}K_{\text {RBF }} \text { kernel } \\
\text { function }\end{array}$ & $(48,45,42)$ & $(84 \%, 85 \%$, & $(1.376 \mathrm{~s}$, \\
\hline
\end{tabular}




\begin{tabular}{|c|c|c|c|c|}
\hline $\begin{array}{c}\text { CS-LBP } \\
\text { feature }(\mathrm{N}=2, \\
4,6)\end{array}$ & & & & $1.397 \mathrm{~s})$ \\
\hline Block two & $K_{H I K}$ kernel & $(15,15,12)$ & $(95 \%, 95 \%$, & $(1.501 \mathrm{~s}$, \\
dimensional & function & & $96 \%)$ & $1.521 \mathrm{~s}$, \\
CS-LBP & & & $1.557 \mathrm{~s})$ \\
feature (N=2, & & & & \\
$4,6)$ & & & & \\
\hline
\end{tabular}

As can be seen from the results on the table, the recognition rate of the algorithm improve a lot forORL database. Because the image is not predictable, so more and more face recognition tasks will be carried out in different directions of the human face database, this algorithm improves the recognition rate of about $9 \%$ on this database. Due to the introduction of 2D CS-LBP texture features, the classification and recognition rate of the data set for the change of the human face angle has been improved. At the same time, it can reduce the number of iterations to solve the SVM classification results, and the time complexity of the algorithm is also reduced to a certain extent.

For the Yale database, the improved algorithm of the anti-noise ability is weaker. Compared with the traditional algorithm, this algorithm is not enough to improve the correct rate of face recognition under the illumination change, which can only improve the recognition rate of $3 \%$.

\section{Conclusions}

This paper analyzes the shortcomings of the traditional face recognition algorithm based on LBP feature and SVM classifier, and puts forward block two dimensional CS-LBP texture feature with local and global information. In the choice of the kernel function of the classifier, histogram cross kernel function is adopted. The method is effective in the recognition of the histogram feature, and can improve the classification efficiency and reduce the time complexity. Subsequently, the experiments were performed on the ORL and Yale databases. The experimental results show that the algorithm can improve the correct rate of face recognition in a certain degree based on the ORL database. In addition, the enhancement effect of this algorithm is not obvious on the ORL database. The face recognition algorithm under the influence of noise is the direction of future efforts.

\section{Acknowledgement}

This work was supported by the Guangxi province Natural Science Foundation of China under Grant No. 2014GXNSFAA118392, Educational Commission of Guangxi province Grant No. YB2014209.

\section{References}

[1] J. Li, M. Dong, A.Ren, J. Ren, J. Zhang and L. Huang, "Structural attributes of the temporal lobe predict face recognition ability in youth", Neuropsychologia, (2016).

[2] S. Wang, J. Lu, X. Gu, B. A. Weyori and J. Y. Yang, "Unsupervised discriminant canonical correlation analysis based on spectral clustering”, Neurocomputing, vol. 171, (2016).

[3] F. Škanta, P. Procházková, R. Roubalová, J. Dvořák and M. Bilej, "LBP/BPI homologue in Eiseniaandreiearthworms", Developmental and Comparative Immunology, vol. 541, (2016).

[4] W. H. Lin, P. Wang and C. F. Tsai, "Face recognition using support vector model classifier for user authentication", Electronic Commerce Research and Applications, (2016).

[5] D. Wang, J. Yang, J. Deng and Q. Liu, "FaceHunter : A multi-task convolutional neural network based face detector", Signal Processing: Image Communication, (2016).

[6] J. Jin, B. Xu, X. Liu, Y. Wang, L. Cao, L. Han, B. Zhou and M. Li, "A face detection and location method based on Feature Binding”, Signal Processing: Image Communication, , vol. 36, (2015). 
[7] C. Qin, X. Chen, D. Ye, J. Wang and X. Sun, "A Novel Image Hashing Scheme with Perceptual Robustness Using Block Truncation Coding”, Information Sciences, (2016).

[8] D. Bhattacharjee, D. K. Basu, M. Nasipuri and M. Kundu, "Human face recognition using fuzzy multilayer perceptron", Soft Computing, vol. 146, (2010).

[9] J. J. Ye and J. Zhang, "Enhanced Karush-Kuhn-Tucker condition and weaker constraint qualifications", Mathematical Programming, vol. 1391, (2013).

[10] Y. Xu, Z. Li, J. S. Pan and J. Y. Yang, "Face recognition based on fusion of multi-resolution Gabor features", Neural Computing and Applications, , vol.235, (2013).

[11] L. Wang, R. F. Li, K. Wang and J. Chen, "Feature representation for facial expression recognition based on FACS and LBP", International Journal of Automation and Computing, vol. 115, (2014).

[12] H. Jingwen, C. C. Clifford, H. Y. King and I. K. Albert, "A weighted logistic regression analysis for predicting the odds of head/face and neck injuries during rollover crashes", Annual proceedings, vol. 51, (2008).

[13] S. Ganesh Vaidyanathan, Bibhas Kar, N. Kumaravel, "A regression based approach to a maximum margin classifier for separation of linearly inseparable pattern classes", Journal of Interdisciplinary Mathematics, vol. 112, (2008).

[14] W. Jianxin, "Efficient HIK SVM learning for image classification", IEEE Transactions on Image Processing, vol. 2110, (2012).

[15] Anonymous. Yale University; "Women with high blood pressure during pregnancy face future of complications", NewsRx Health \&amp; Science, (2009).

\section{Authors}

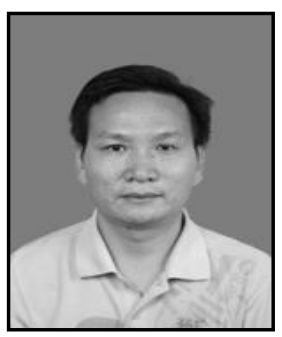

Shaoming Pan, he is anassociate professor of School of Electrical and Information Engineering in Guangxi University of Science and Technology. He has got the Master Degree.

Gongkun Luo, he is a lecturer of School of Electrical and Information Engineering in Guangxi University of Science and Technology. He has got the Master Degree. 
International Journal of Signal Processing, Image Processing and Pattern Recognition Vol. 9, No. 12, (2016) 